\title{
Inpatient burden of esophageal varices in the United States: analysis of trends in demographics, cost of care, and outcomes
}

\author{
Shantanu Solanki ${ }^{1}$, Khwaja Fahad $\mathrm{Haq}^{2}$, Raja Chandra Chakinala ${ }^{3}$, Zubair Khan ${ }^{4}$, Wilbert S. Aronow ${ }^{5}$, \\ Muhammad Ali Khan ${ }^{6}$, Mohamed Tausif Siddiqui ${ }^{3}$, Khwaja Saad Haq ${ }^{7}$, Shalom Frager ${ }^{8}$, Maryam Alimirah', \\ Christopher Nabors ${ }^{3}$, David J. Samson ${ }^{10}$, Edward Lebovics ${ }^{8}$, David Cary Wolf ${ }^{8}$
}

${ }^{1}$ Hospitalist Department, Guthrie Robert Packer Hospital, Sayre, PA, USA; ${ }^{2}$ Division of Gastroenterology, Henry Ford Hospital, Detroit, MI, USA; ${ }^{3}$ Department of Medicine, New York Medical College at Westchester Medical Center, Valhalla, NY, USA; ${ }^{4}$ Department of Gastroenterology, Hepatology \& Nutrition, The University of Texas Health Science Center at Houston, Houston, TX, USA; ${ }^{5}$ Department of Cardiology, New York Medical College at Westchester Medical Center, Valhalla, NY, USA; ${ }^{6}$ Division of Gastroenterology, University of Tennessee Health Science Center, Memphis, TN, USA; ${ }^{7}$ Department of Medicine, Kingsbrook Jewish Medical Center, Brooklyn, NY, USA; ${ }^{8}$ Division of Gastroenterology \& Hepatobiliary Diseases, New York Medical College at Westchester Medical Center, Valhalla, NY, USA; ${ }^{9}$ Department of Medicine, Henry Ford Hospital, Detroit, MI, USA; ${ }^{10}$ Clinical Research Unit, Department of Surgery, Westchester Medical Center, Valhalla, NY, USA

Contributions: (I) Conception and design: S Solanki, KF Haq, DC Wolf; (II) Administrative support: S Solanki, KF Haq, WS Aronow, DC Wolf; (III) Provision of study materials or patients: S Solanki; (IV) Collection and assembly of data: S Solanki, DJ Samson; (V) Data analysis and interpretation: S Solanki, DJ Samson, KF Haq, RC Chakinala, M Alimirah, DC Wolf; (VI) Manuscript writing: All authors; (VII) Final approval of manuscript: All authors.

Correspondence to: Shantanu Solanki, MD, MPH. Hospitalist Department, Guthrie Robert Packer Hospital, 211B W Lockhart Street, Sayre, PA 18840, USA. Email: shan4788@gmail.com.

Background: Esophageal variceal bleeding remains a common reason for hospitalization in the United States. The main objective of this study was to analyze demographic variations and outcomes in hospitalizations related to esophageal varices (EV) in the US.

Methods: We performed a retrospective observational cohort study using National Inpatient Sample (NIS) database for all hospitalizations with discharge diagnoses of EV, with and without hemorrhage from 2001 to 2011 .

Results: In 2001, there were 19,167 hospitalizations with discharge diagnoses of EV with and without bleeding compared to 45,578 in $2011(\mathrm{P}<0.001)$. There was a $138 \%$ increase in the number of total EV hospitalizations, a 221\% increase in hospitalizations with $\mathrm{EV}$ without hemorrhage, and a $7 \%$ increase in hospitalizations for patients with EV and hemorrhage. Age group 50-64 was the most affected, accounting for $31.4 \%$ of $\mathrm{EV}$ hospitalizations in 2001 and $46.7 \%$ of $\mathrm{EV}$ hospitalizations in $2011(\mathrm{P}<0.001)$. The overall in-hospital mortality rate was 3.4\% for patients with $\mathrm{EV}$ without hemorrhage and $8.7 \%$ for patients with $\mathrm{EV}$ with hemorrhage $(\mathrm{P}=0.0003)$.

Conclusions: The number of hospitalizations for patients with asymptomatic EV increased significantly between 2001 to 2011, with only a small concurrent increase in the number of hospitalizations for patients with esophageal variceal bleeding.

Keywords: Esophageal varices (EV); cirrhosis; endoscopic treatment

Submitted Mar 11, 2019. Accepted for publication Aug 07, 2019.

doi: 10.21037/atm.2019.08.34

View this article at: http://dx.doi.org/10.21037/atm.2019.08.34 


\section{Introduction}

The prevalence of cirrhosis is increasing at an alarming rate in the U.S., to a great extent being driven by the increased number of cases of nonalcoholic fatty liver disease (NAFLD) and nonalcoholic steatohepatitis (NASH) $(1,2)$. The prevalence of NAFLD-associated advanced fibrosis and NASH-associated cirrhosis increased 2-fold and 2.5-fold, respectively, between the years 1999-2002 and 2009-2012 (3). In addition, the number of patients presenting with $\mathrm{HCV}$-related cirrhosis is predicted to double over the next twenty years (4).

Cirrhosis is the twelfth leading cause of death in the U.S. (5). Cirrhosis accounted for 37,890 deaths in 2013 (5). One study estimated that the annual incidence of cirrhosisrelated deaths would increase to 60,000 deaths if diagnostic codes for viral hepatitis and cirrhosis complications were also considered (6). As a result, cirrhosis poses a large economic burden. One study estimated the annual cost of care of patients with cirrhosis to be about $\$ 2$ billion (7).

Esophageal variceal bleeding is a common complication of cirrhosis. Nearly $30 \%$ of patients with esophageal varices (EV) bleed within the first year after diagnosis (8). Patients hospitalized for esophageal variceal bleeding are at significantly increased risk of in-hospital mortality, compared to patients admitted with non-bleeding varices. Currently, the estimated burden of treating bleeding EV remains unknown. Accordingly, an accurate understanding of trends in EV-related hospitalizations is necessary for appropriate healthcare planning. This study was therefore undertaken to identify vulnerable groups and the impact of variceal bleeding within a nationally representative patient sample (9).

\section{Methods}

\section{Source of data}

The National Inpatient Sample (NIS), designed by Agency for Healthcare Research and Quality (AHRQ), is the largest all-payer inpatient database in the U.S. This data is compiled yearly and contains discharge data from over 1,200 hospitals located across 45 states in the U.S. The NIS was designed to approximate a $20 \%$ stratified sample of community hospitals in the country and provides sampling weights to calculate national estimates (10). The NIS contains information included in a typical discharge summary, with safeguards in place to protect the privacy of individual patients, physicians, and hospitals. Each individual hospitalization is de-identified and is maintained in the NIS as a unique entry with one primary discharge diagnosis and approximately 24 secondary diagnoses during that hospitalization. Each entry also carries information on demographic details, insurance status, comorbidities, primary/secondary procedures, hospitalization outcomes, length of stay, and cost of care. The internal validity of the database is guaranteed by annual data quality assessments of the sample. Moreover, comparisons with data sources like the American Hospital Association (AHA) Annual Survey of Hospitals, the National Hospital Discharge Survey from the National Center for Health Statistics, and the Medicare Provider and Analysis Review (MedPAR) inpatient data from the Centers for Medicare and Medicaid Services strengthen the external validity of the sample $(11,12)$.

\section{Study design}

We queried the NIS database from year 2001 to 2011 using a retrospective observational cohort study design to identify all hospitalizations with EV. We extracted data regarding all of the hospitalizations from 2001 to 2011 with primary or secondary diagnosis of $\mathrm{EV}$ with hemorrhage and without hemorrhage, which in turn were identified with validated International Classification of Diseases, 9th Revision, Clinical Modification (ICD-9-CM) codes 456.0 and 456.1, respectively. Patients with age less than 18 years were excluded. Also, all hospitalizations with missing information related to age, gender, admission/discharge date, and inhospital mortality status were excluded to gather and document accurate demographics of EV hospitalizations. To calculate the estimated cost of hospitalizations, the NIS data were merged with cost-to-charge ratio (CCR) files available from the Healthcare Cost and Utilization Project. We estimated the cost of each inpatient stay by multiplying the total hospital charge with the CCR.

\section{Variables and statistical analysis}

SAS 9.4 (SAS Institute Inc., Cary, NC, USA) and Joinpoint Trend Analysis Software (version 4.5.0.1) were utilized for complex statistical analyses. Since NIS represents a $20 \%$ stratified random sample of U.S. hospitals, analyses were performed using hospital-level discharge weights provided by the NIS, to obtain national estimates of hospitalizations. Community hospitals are oversampled as the NIS only includes data from community hospitals. The NIS is sampled from the State Inpatient Databases (SID), which 


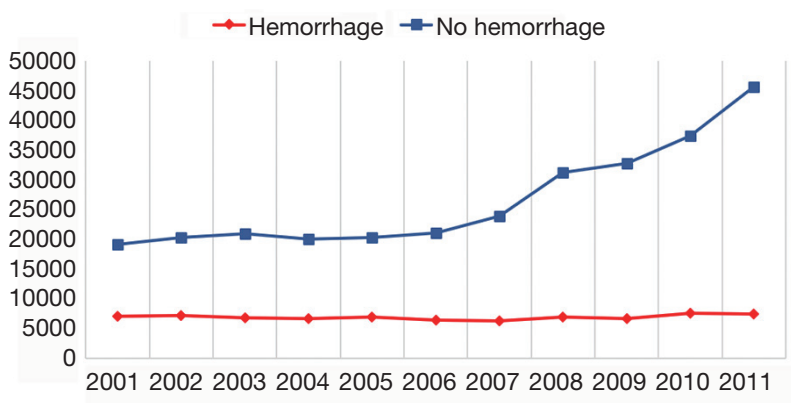

Figure 1 Trend in number of EV hospitalizations with and without hemorrhage. EV, esophageal varices.

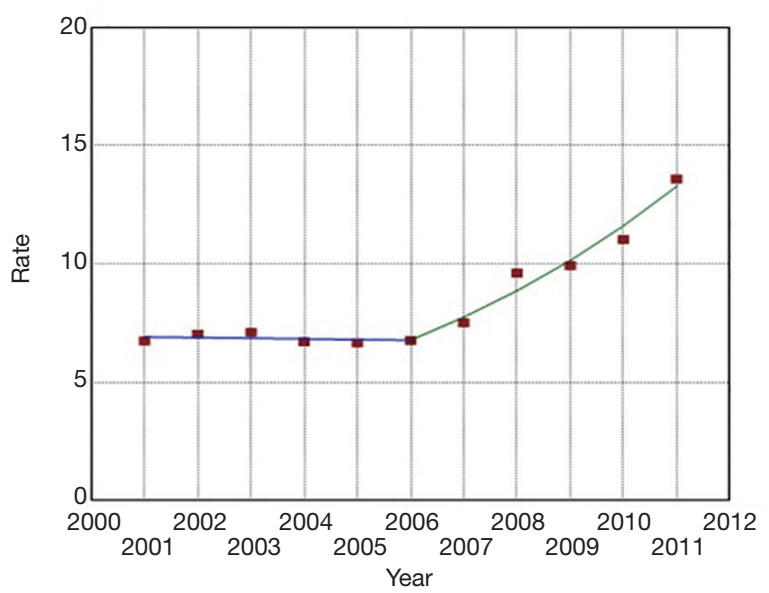

Figure 2 APC in number of hospitalizations. APC, annual percent change.

captures hospital inpatient stays in a given state. The SID encompasses about $97 \%$ of all U.S. community hospital discharges. EV-related hospitalizations per million U.S. population were calculated by dividing the number of such hospitalizations in each year by the U.S. census population greater than 18 years age for that year. EV hospitalizations were also calculated in subgroups of age (18-34, 35-49, $50-64,65-79$, and $\geq 80$ years), gender, race (white, black, Hispanic, and other), insurance status (Medicare/Medicaid, private insurance, and self-pay/other), hospital location in different U.S. regions (Northeast, Midwest, South, and West), and teaching status of the hospital. According to AHRQ, a hospital is considered to be a teaching hospital if it is: (I) an AMA-approved residency program, (II) a member of the Council of Teaching Hospitals, or (III) a hospital with a full-time resident-to-bed ratio more than 0.25 (13). The Cochrane-Armitage trend test was used to calculate trends in categorical variables (14). The Wilcoxon rank sum test was used to assess continuous variables (15). To calculate the annual percent change (APC) in the number of hospitalizations, we obtained the age-adjusted crude hospitalization rate for each year and then multiplied it by the age distribution of the standard population for that year. Finally, Joinpoint regression was utilized to obtain APC values (16).

\section{Results}

\section{EV hospitalizations and demographics}

A total of 292,851 hospitalizations for EV (as a primary or secondary discharge diagnosis) were identified in the U.S. population from 2001 to 2011. (Figure 1). EV hospitalizations increased by $138 \%$ from 19,167 in 2001 to 45,578 in $2011(\mathrm{P}<0.001)$. The number of U.S. hospitalizations with an $\mathrm{EV}$ diagnosis was relatively stable between 2001 and 2006. However, beginning in 2006, there was a significant increase in the percent of U.S. hospitalizations with a diagnosis of EV. (Figure 2). Patient characteristics are described in Table $1 . \mathrm{EV}$ patients were predominantly white $(53 \%)$ and between 50 and 64 years of age $(40.7 \%)$. The second most commonly affected race was Hispanic (14.6\%). Similar racial distribution was seen in the EV patients in a study conducted by Jamal et al. (17). There were more hospitalizations in males $(63.8 \%)$ than in females $(36.2 \%)$. The gender ratio was stable throughout the study period. Medicare/Medicaid was the primary payer for $59.5 \%$ of EV hospitalizations. A plurality of EV hospitalizations were reported in the South $(37.5 \%)$, followed by the Midwest (22.7\%), West (22.5\%), and Northeast (17.4\%) (Table 1). However, the incidence of EV hospitalizations was highest in the West, accounting for 84 hospitalizations per 100,000 hospitalizations when compared to 74 per 100,000 hospitalizations in the South.

\section{Trends in EV hospitalizations}

The EV hospitalization rate more than doubled between 2001 and 2011, increasing from 515 to 1,181 per million U.S. population per year $(\mathrm{P}<0.001)$ (Table 2). Over the same period, the rate of hospitalization for $\mathrm{EV}$ without hemorrhage increased from 326 to 990 per million U.S. population per year, an increase of $203 \%$. The rate of hospitalization for $\mathrm{EV}$ with hemorrhage increased from 189 to 191 per million U.S. population per year, an increase 


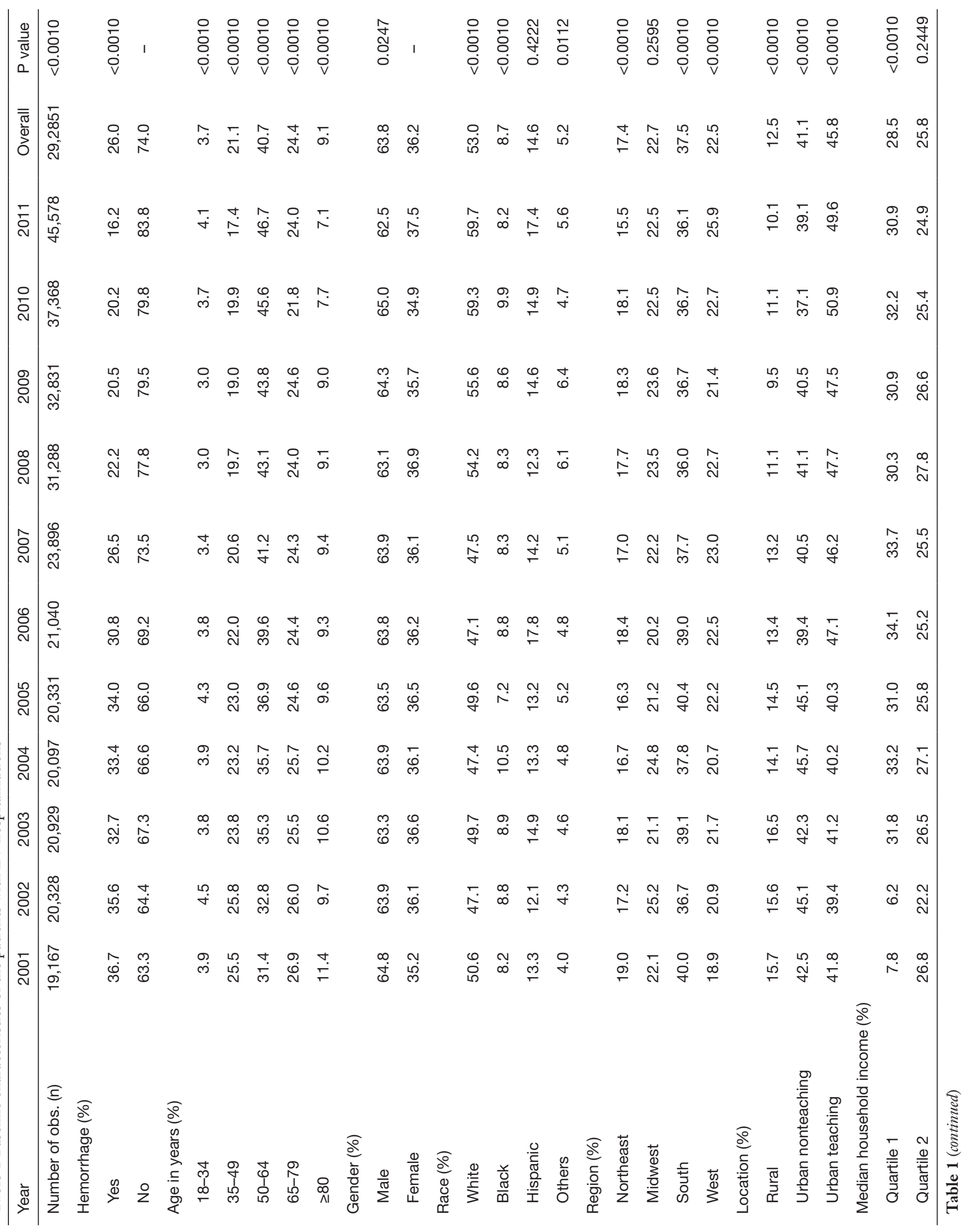




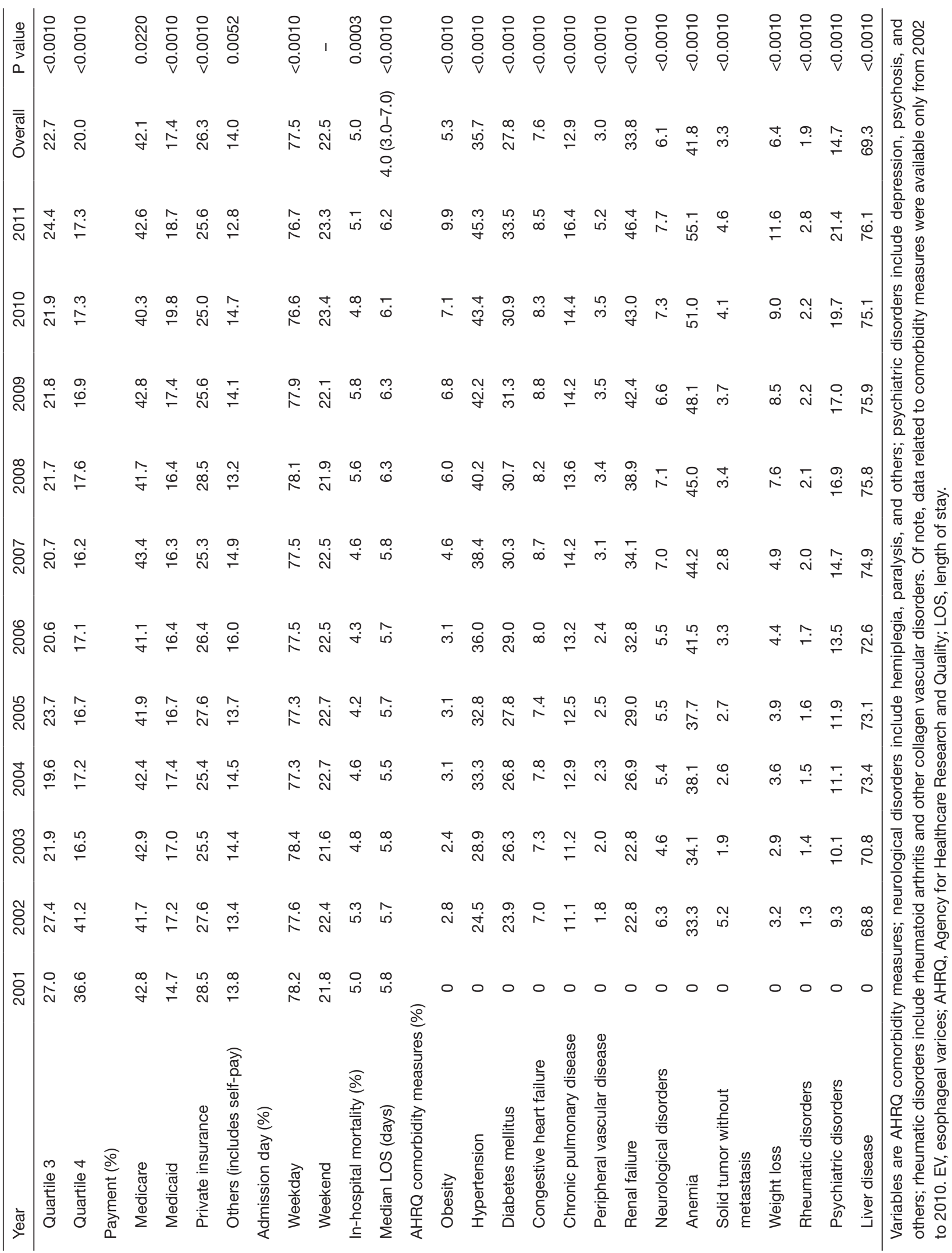


Table 2 EV hospitalization/1 million U.S. population

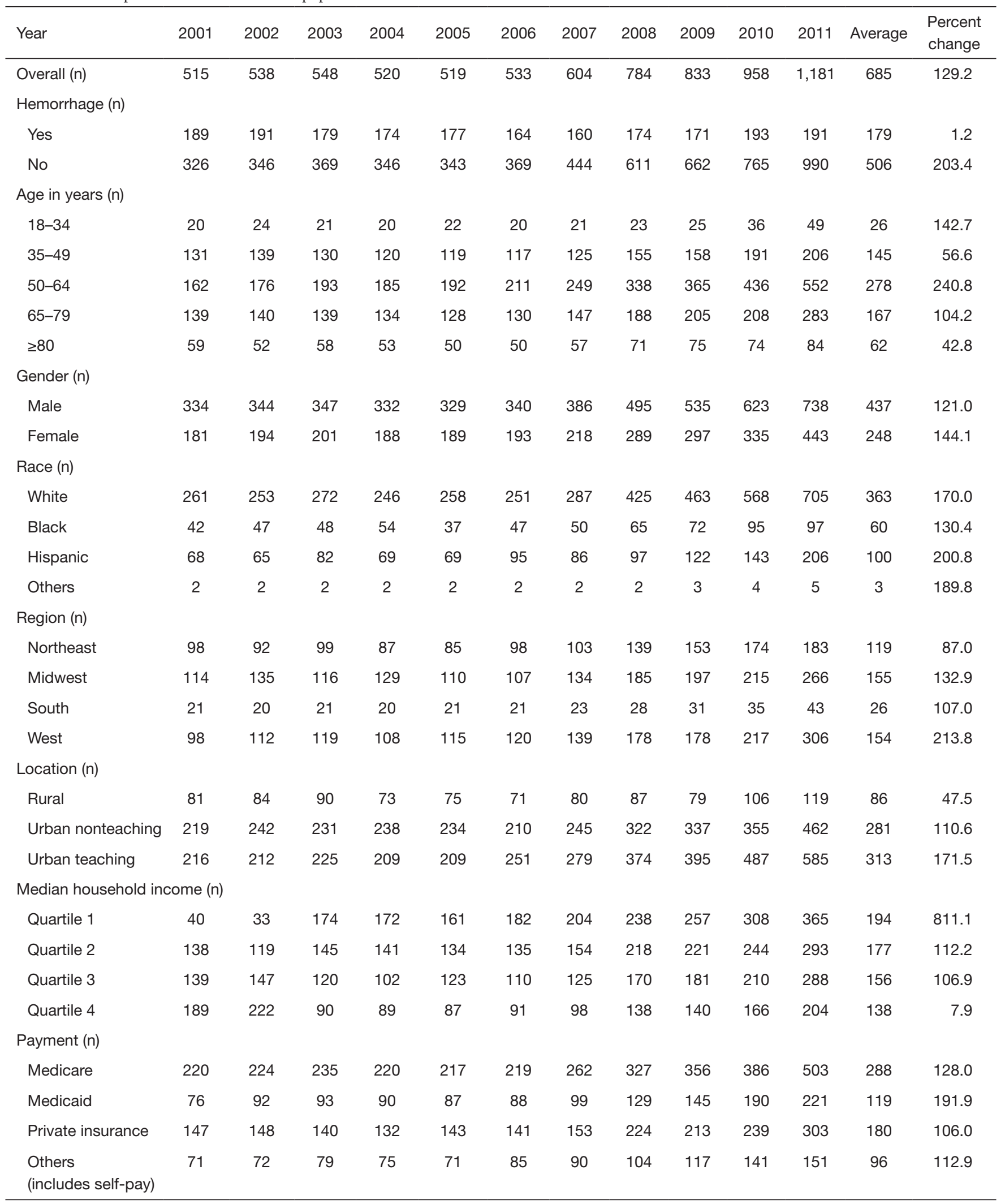

EV, esophageal varices. 
of $1.2 \%$. The number of hospitalizations for $\mathrm{EV}$ with hemorrhage increased only $7 \%$ over the decade, from 7,036 hospitalizations in 2001 to 7,451 hospitalizations in 2011 .

The hospitalization rate increased across all age groups. The magnitude of rise in EV-related hospitalizations was highest in the 50-64 age group (relative increase 240.8\%, $\mathrm{P}<0.001$ ) and lowest in $>80$ years age group (relative increase $42.8 \%, \mathrm{P}<0.001$ ) (Table 2).

The EV hospitalization rate was higher in white males than other racial groups throughout the study. The EV hospitalization rate for whites was 261 per million U.S. population in 2001, increasing to 705 per million U.S. population in 2011 , a $170 \%$ increase. Hispanics saw a greater surge in EV hospitalizations, with an EV hospitalization rate of 68 per million U.S. population in 2001, increasing to 206 per million U.S. population in 2011, a $201 \%$ increase $(\mathrm{P}<0.001)$.

The EV hospitalization rate for men and women were 334 and 181, respectively, per million U.S. population in 2001. The hospitalization rates increased to 738 and 443, respectively, per million U.S. population in 2011. This amounted to a $121 \%$ increase for men and a $144 \%$ increase for women $(\mathrm{P}<0.001)$.

\section{All-cause in-bospital mortality}

The mortality rate for patients hospitalized for $\mathrm{EV}$ without hemorrhage increased from $2.6 \%$ to $4.3 \%$ between 2001 and $2011(\mathrm{P}<0.001)$. Not unexpectedly, the mortality rate for patients hospitalized for $\mathrm{EV}$ with hemorrhage was higher. However, magnitude of the rise was not as dramatic, going from a mortality rate of $9.2 \%$ in 2001 to $9.6 \%$ in $2011(\mathrm{P}<0.001)$ (Table 3).

Across the age groups, the mortality rate was highest in $>80$ years age group at $5.5 \%$. The mortality rate was slightly higher in males $(5.3 \%)$, non-whites and other races $(5.9 \%)$, and patients with Medicaid insurance (5.6\%) (Table 3).

\section{Length of stay and regional differences in cost of care}

The median LOS was 4 days with an interquartile range of 2-7 days $(\mathrm{P}=0.0002)$ (Figure 3). After adjustment for inflation, the mean cost of hospitalizations with an $\mathrm{EV}$ diagnosis increased from $\$ 11,274$ in 2001 to $\$ 15,160$ in $2011(\mathrm{P}<0.001)$ (Figure 4). The mean cost of hospitalization was $\$ 12,322$ for $\mathrm{EV}$ without hemorrhage, compared to $\$ 15,202$ for $\mathrm{EV}$ and hemorrhage $(\mathrm{P}<0.001)$ (see Table 4). We estimate the annual national cost of managing patients with an EV diagnosis to have been \$216 million in 2001 and $\$ 690$ million in 2011. The mean cost of care was lowest if EV-related hospitalization was in the Northeast $(\$ 4,628)$ and rural hospitals $(\$ 3,337)$. It was highest in the South $(\$ 9,983)$ and urban teaching hospitals $(\$ 12,179)$ (Table 4).

\section{Comorbidities associated with EV-related hospitalizations}

As per AHRQ comorbidity measures, EV-related hospitalizations were most commonly associated with anemia (41.8\%), followed by hypertension $(35.6 \%)$ and renal disease $(33.8 \%)$. Up to $15 \%$ of hospitalizations with $\mathrm{EV}$ were associated with psychiatric disorders (Table 1). Patients with EV and hemorrhage were found to have more comorbidities compared to patients with $\mathrm{EV}$ without hemorrhage (Table 5).

\section{Discussion}

Our study reports important findings associated with EVrelated hospitalizations over an 11-year period in the U.S. There was a significant increase in the total number of EVrelated hospitalizations over the study period. However, the increase in the number of cases of $\mathrm{EV}$ with hemorrhage was much lower than the increase in the number of cases of $\mathrm{EV}$ without hemorrhage. We postulate that this may be due to improved surveillance of patients at risk for having EV. Current guidelines recommend the performance of esophagogastroduodenoscopy every $2-3$ years in patients without a prior history of EV and every 1-2 years in patients with small varices (18). It is possible that the implementation of these recommendations in practice is leading to: (I) increased diagnosis of patients with $\mathrm{EV}$; (II) increased use of prophylactic strategies to prevent bleeding, like beta blocker therapy and endoscopic variceal ligation. It is also possible that hospitals are employing more thorough coding techniques as they review charts for billing purposes.

Hospitalizations for patients with $\mathrm{EV}$ with hemorrhage were associated with more comorbidities and higher costs that hospitalizations for patients with $\mathrm{EV}$ without hemorrhage. Not surprisingly, EV hospitalizations with hemorrhage had a higher mortality rate than those without hemorrhage. Interestingly, the overall mortality rate of $8.7 \%$ for the study period was much lower than the approximate $20 \%$ mortality rate that is typically quoted in discussions of variceal bleed management (19-21). This potentially reflects inaccuracies in the overall coding system.

Surprisingly, our data showed rising rates of mortality 
Table 3 In-hospital mortality for EV hospitalization

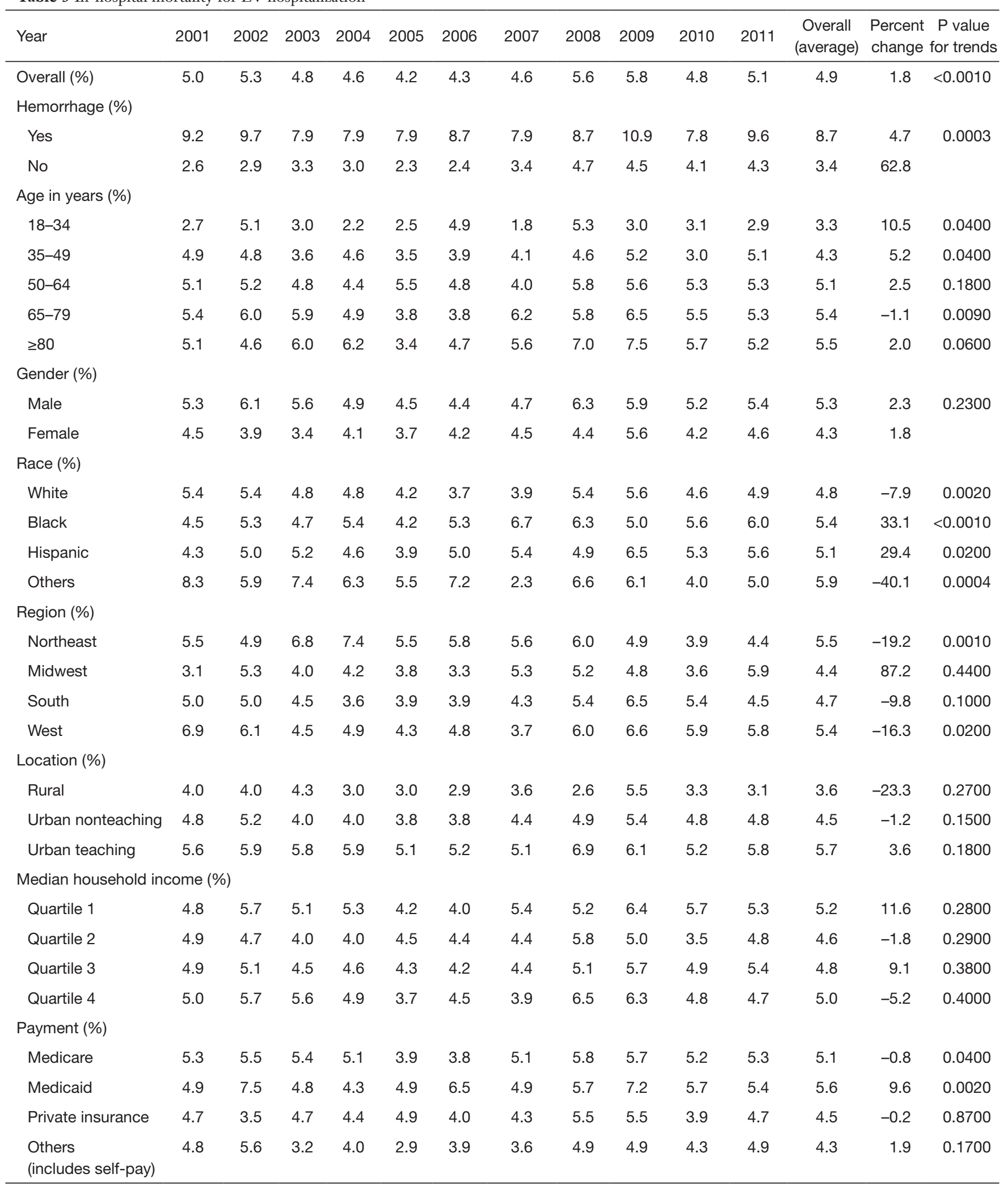

EV, esophageal varices. 


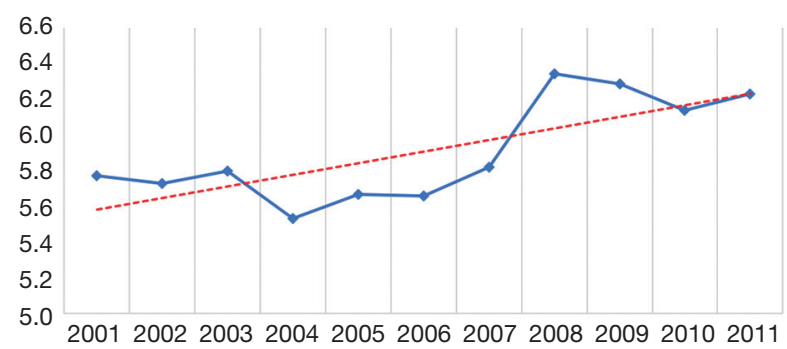

Figure 3 Trends in median LOS and in-hospital mortality. LOS, length of stay.

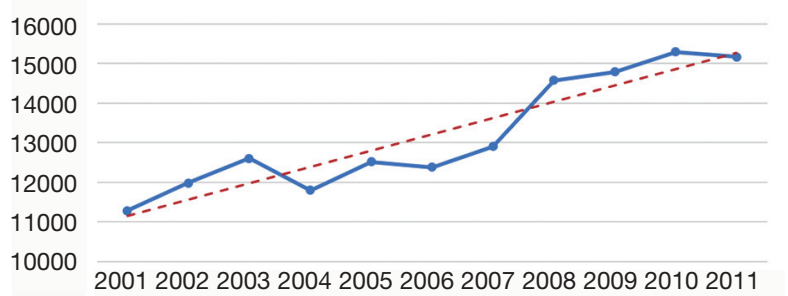

Figure 4 Trends in cost of care for EV hospitalizations. EV, esophageal varices.

for patients with $\mathrm{EV}$ without and with hemorrhage between 2001 and 2011. This finding could reflect changes in diagnostic coding. Alternatively, it could reflect the fact that hospitals are caring for older cirrhotic patients with more comorbidities.

Most of the hospitalizations in our study were identified in the 50-64 years age group; the fewest were in the 18-34 years age group. This finding correlates with what is felt to be an aging U.S. population of patients with cirrhosis. It may be attributed to the development of NAFLD in an older population of individuals with obesity and diabetes, and due to the aging of persons who were originally infected with hepatitis C in the 1970s and 1980s.

The number of hospitalizations for cirrhosis is steadily increasing in the U.S. One study recorded 337,956 cirrhosis hospitalizations in 2002, as compared to 570,220 cirrhosis hospitalizations in 2012 (22). However, the data is conflicting regarding trends in hospitalizations for esophageal variceal bleeding (EVB). Jamal et al. reported a decline in EVB hospitalizations from 1988 to 2002 (17). This does not correlate with data that we analyzed. This is likely because of better implementation of variceal screening during our study period. Pant et al. also reported a decrease in the incidence of EVB, for the period from 2002 until

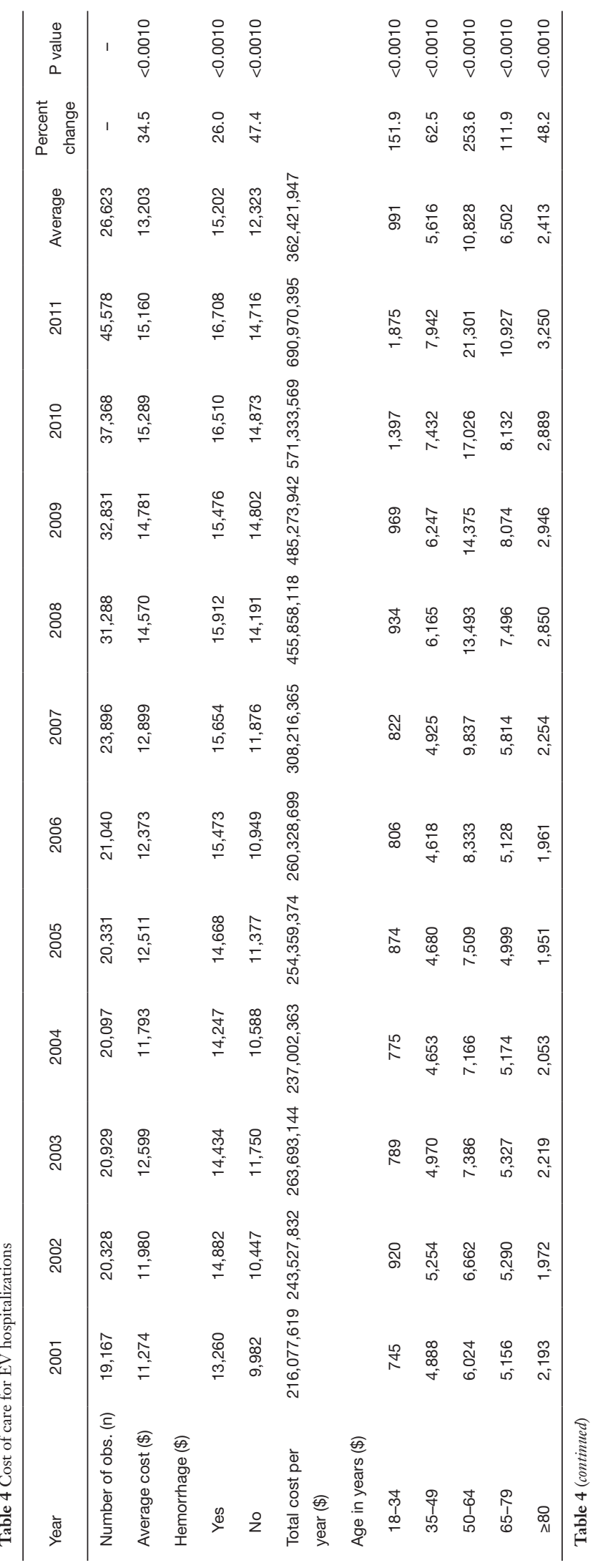




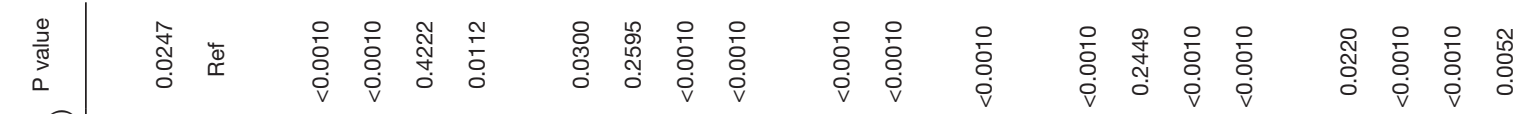

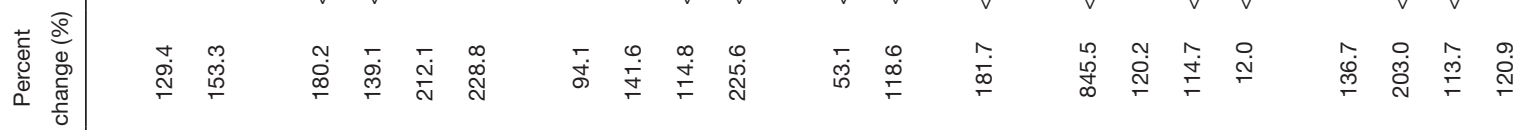

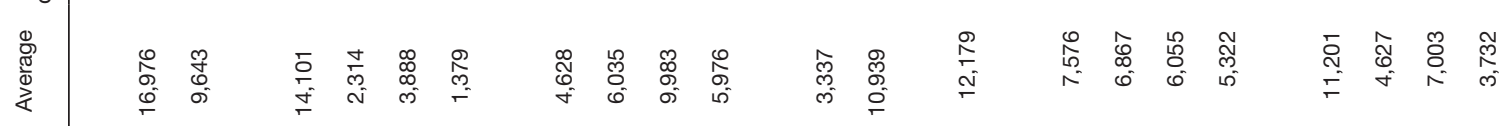

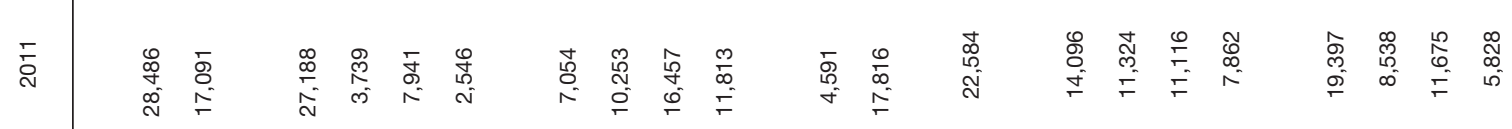

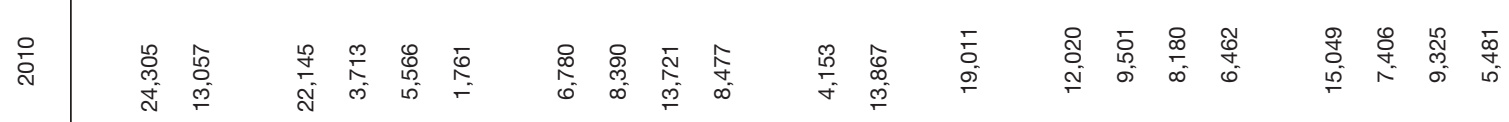

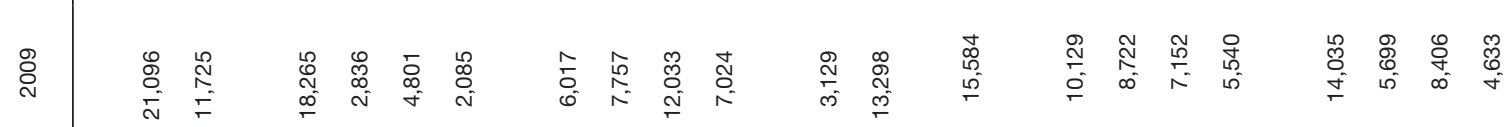

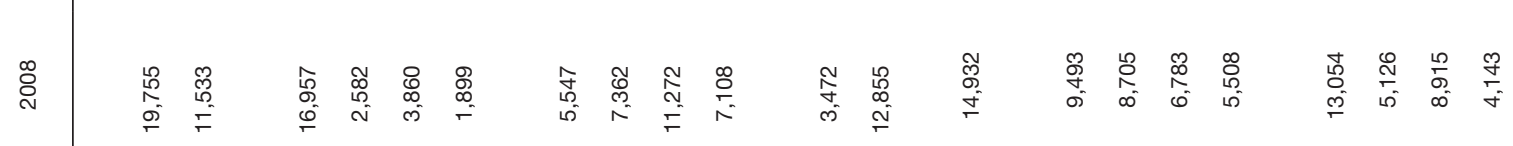

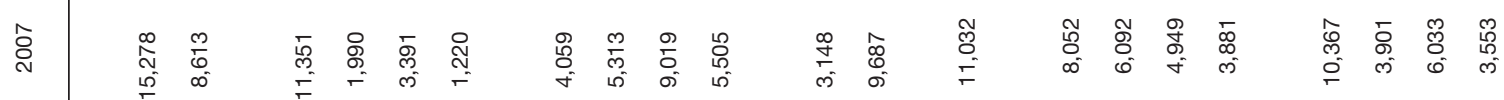

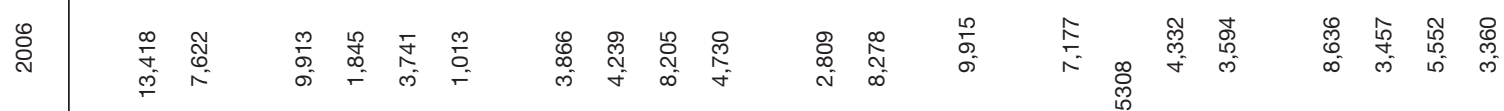

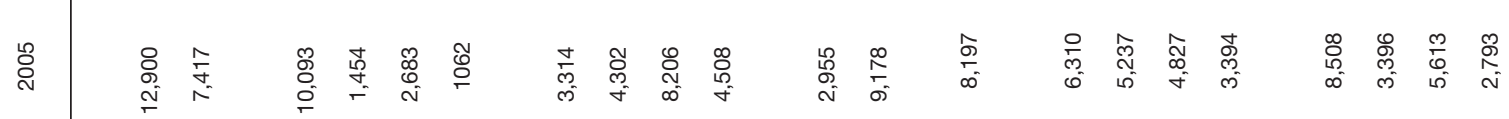

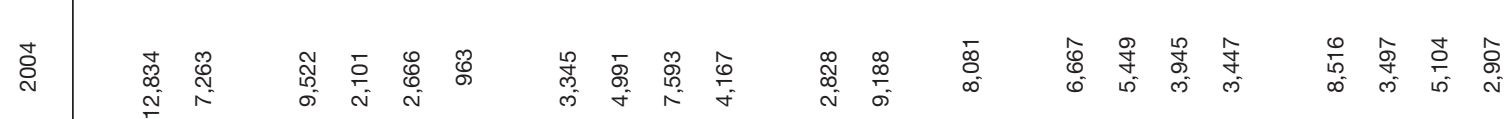

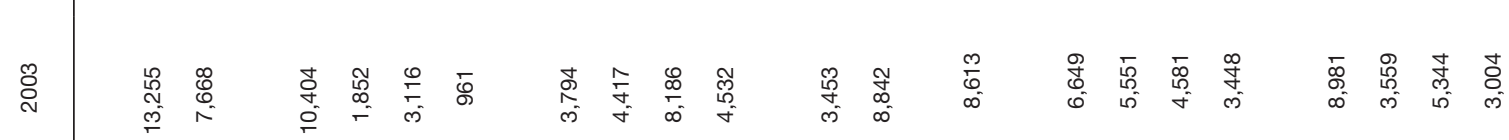

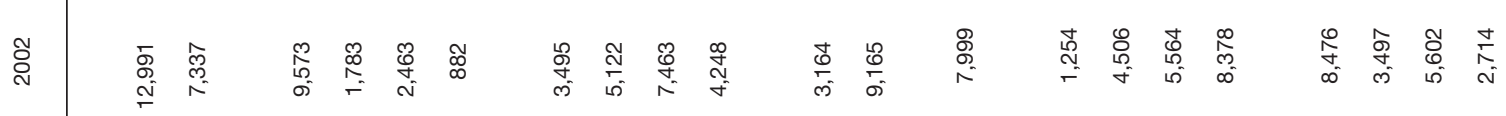

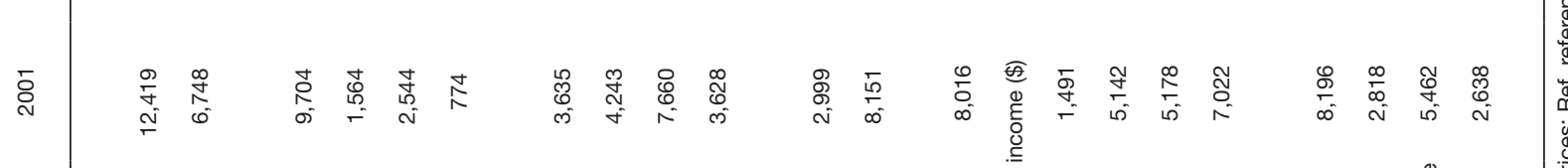

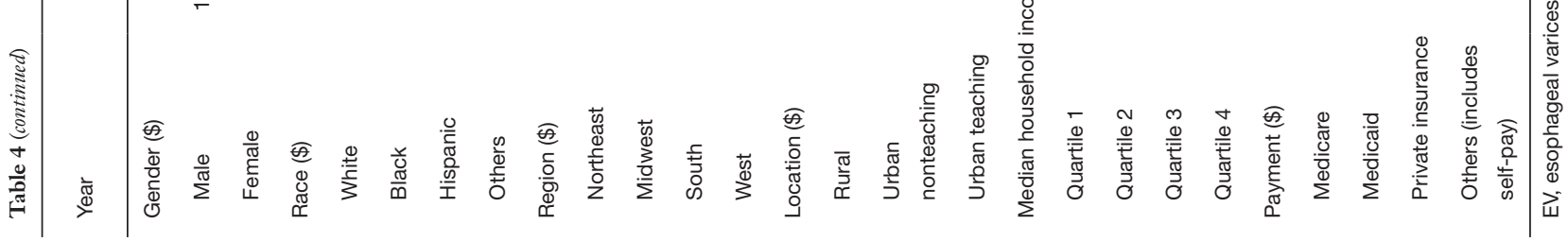


Table 5 Predictors of bleeding for EV hospitalizations

\begin{tabular}{|c|c|c|c|c|}
\hline Variables & Odds ratio & \multicolumn{2}{|c|}{$95 \%$ confidence limits } & $P$ value \\
\hline $18-34$ & Referent & & & \\
\hline $35-49$ & 1.08 & 0.97 & 1.21 & 0.170 \\
\hline $50-64$ & 0.94 & 0.85 & 1.05 & 0.290 \\
\hline $65-79$ & 0.82 & 0.73 & 0.92 & $<0.001$ \\
\hline$\geq 80$ & 0.81 & 0.71 & 0.93 & $<0.001$ \\
\hline \multicolumn{5}{|l|}{ Race (\%) } \\
\hline White & Referent & & & \\
\hline Black & 1.13 & 1.05 & 1.21 & $<0.001$ \\
\hline Hispanic & 1.24 & 1.14 & 1.36 & $<0.001$ \\
\hline Others & 1.21 & 1.08 & 1.35 & $<0.001$ \\
\hline \multicolumn{5}{|l|}{ Region (\%) } \\
\hline Northeast & Referent & & & \\
\hline Midwest & 1.05 & 0.98 & 1.14 & 0.180 \\
\hline South & 1.33 & 1.25 & 1.41 & $<0.001$ \\
\hline West & 1.23 & 1.15 & 1.31 & $<0.001$ \\
\hline \multicolumn{5}{|l|}{ AHRQ comorbidity measures (\%) } \\
\hline AIDS & 1.44 & 1.16 & 1.77 & $<0.001$ \\
\hline Alcohol abuse & 1.27 & 1.21 & 1.33 & $<0.001$ \\
\hline Deficiency anemias & 0.76 & 0.72 & 0.81 & $<0.001$ \\
\hline Rheumatic disorders & 1.17 & 1.01 & 1.35 & 0.040 \\
\hline Chronic blood loss anemia & 2.20 & 2.07 & 2.35 & $<0.001$ \\
\hline Coagulopathy & 1.13 & 1.07 & 1.18 & $<0.001$ \\
\hline Depression & 0.66 & 0.61 & 0.72 & $<0.001$ \\
\hline Diabetes mellitus & 1.16 & 1.10 & 1.22 & $<0.001$ \\
\hline Hypertension & 0.87 & 0.83 & 0.91 & $<0.001$ \\
\hline Lymphoma & 1.26 & 1.01 & 1.58 & 0.040 \\
\hline Fluid and electrolyte disorders & 0.95 & 0.90 & 1.00 & 0.030 \\
\hline Metastatic cancer & 1.73 & 1.53 & 1.94 & $<0.001$ \\
\hline Obesity & 0.77 & 0.70 & 0.85 & $<0.001$ \\
\hline Peripheral vascular disorders & 0.76 & 0.66 & 0.87 & $<0.001$ \\
\hline Psychiatric disorder & 0.77 & 0.68 & 0.86 & $<0.001$ \\
\hline Pulmonary circulation disorders & 0.64 & 0.54 & 0.76 & $<0.001$ \\
\hline Renal failure & 0.80 & 0.74 & 0.87 & $<0.001$ \\
\hline Weight loss & 0.80 & 0.73 & 0.88 & $<0.001$ \\
\hline
\end{tabular}

EV, esophageal varices; AHRQ, Agency for Healthcare Research and Quality; AIDS, acquired immune deficiency syndrome. 
2012 (22). However, their study included only cirrhotic patients with EVB. They did not include hospitalizations of EVB secondary to other causes like portal vein thrombosis and splenic vein thrombosis. Our study reports a $138 \%$ increase in the number of discharges for EV between 2001 and 2011. There was a $221 \%$ increase in the diagnosis of $\mathrm{EV}$ without hemorrhage and a $7 \%$ increase in the diagnosis of $\mathrm{EV}$ with hemorrhage.

There was a significant increase in both the length of stay and cost of care over the study period. This finding is likely due to an aging population and the multiple comorbidities experienced by older patients (23).

Analysis of the NIS database permitted us to study a large population. Such an analysis should decrease the inherent bias seen in studies that are confined to a single region or hospital. However, our analysis had a number of significant limitations. Administrative databases are susceptible to errors that arise from coding inaccuracies. The EV diagnosis and the presence of comorbidities were based on the presence of administrative codes. ICD-9CM codes 456.0 and 456.1 were validated for $\mathrm{EV}$ (with and without hemorrhage, respectively) in the administrative database. The database did not permit us to determine which patients assigned a code of "EV with hemorrhage" were hospitalized for the new onset of variceal bleeding, as opposed to patients who had a diagnosis of variceal bleeding in the past.

There is a risk that our analysis could underestimate the number of variceal bleeds each year. If an EV diagnosis accompanied other "serious" conditions-e.g., "cirrhosis" - the latter might be listed as a primary diagnosis, even if a patient's presenting complaint was actually acute variceal bleeding.

Our analysis could also overestimate the number of patients with EV. Indeed, the NIS considers each hospitalization as separate entry. Thus, there is no coding method that can separate index cases from readmissions in the NIS database.

An increase in the readmission rate for patients with decompensated cirrhosis has been reported (24). Such an analysis is beyond the scope of the current study. It is possible that the increase in the incidence of nonbleeding varices that we observed corresponds with an increase in the number of readmissions for patients with decompensated cirrhosis (24).

Unfortunately, the design of the database allowed us to examine in-hospital characteristics only. It did not permit analysis of long-term follow-up of patient outcomes. It did not permit us to study health care utilization in out-patient settings or emergency departments.

In conclusion, our review of hospitalization trends over the last decade found a consistent increase in the number of hospitalizations associated with EV. There was also an increase in the length of stay and cost of care. The most striking finding was the relatively small increase in the number of cases of $\mathrm{EV}$ with hemorrhage compared to the number of cases of $\mathrm{EV}$ without hemorrhage. We believe that improved out-patient care and implementation of variceal screening and prophylaxis measures will help to reduce the number of hospitalizations for variceal bleeding nation-wide. This will further reduce the economic burden of cirrhosis upon the health care system.

\section{Acknowledgments}

None.

\section{Footnote}

Conflicts of Interest: Presented at Digestive Disease Week. McCormick Place, Chicago, IL, USA. May 2017.

Ethical Statement: The authors are accountable for all aspects of the work in ensuring that questions related to the accuracy or integrity of any part of the work are appropriately investigated and resolved.

\section{References}

1. Vuppalanchi R, Chalasani N. Nonalcoholic fatty liver disease and nonalcoholic steatohepatitis: selected practical issues in their evaluation and management. Hepatology 2009;49:306-17.

2. Younossi ZM, Stepanova M, Afendy $M$, et al. Changes in the prevalence of the most common causes of chronic liver diseases in the United States from 1988 to 2008. Clin Gastroenterol Hepatol 2011;9:524-30.e1; quiz e60.

3. Kabbany MN, Conjeevaram Selvakumar PK, Watt K, et al. Prevalence of nonalcoholic steatohepatitis-associated cirrhosis in the United States: an analysis of national health and nutrition examination survey data. Am J Gastroenterol 2017;112:581-7.

4. Davis GL, Alter MJ, El-Serag H, et al. Aging of hepatitis C virus (HCV)-infected persons in the United States: a multiple cohort model of HCV prevalence and disease progression. Gastroenterology 2010;138:513-21.e1-6. 
5. Yoon $\mathrm{YH}, \mathrm{Chen} \mathrm{CM}$. Liver cirrhosis mortality in the United States: national, state, and regional trends, 20002013. Bethesda: National Institute on Alcohol Abuse and Alcoholism (NIAAA), 2016.

6. Scaglione S, Kliethermes S, Cao G, et al. The epidemiology of cirrhosis in the United States: a population-based study. J Clin Gastroenterol 2015;49:690-6.

7. Neff GW, Duncan CW, Schiff ER. The current economic burden of cirrhosis. Gastroenterol Hepatol (N Y) 2011;7:661-71.

8. Review Team, LaBrecque DR, Abbas Z, et al. World Gastroenterology Organisation global guidelines: nonalcoholic fatty liver disease and nonalcoholic steatohepatitis. J Clin Gastroenterol 2014;48:467-73.

9. Patel NJ, Deshmukh A, Pant S, et al. Contemporary trends of hospitalization for atrial fibrillation in the United States, 2000 through 2010: implications for healthcare planning. Circulation 2014;129:2371-9.

10. Healthcare Cost and Utilization Project. Overview of the National (Nationwide) Inpatient Sample (NIS). Available online: https://www.hcup-us.ahrq.gov/nisoverview.jsp

11. Badheka AO, Chothani A, Mehta K, et al. Utilization and adverse outcomes of percutaneous left atrial appendage closure for stroke prevention in atrial fibrillation in the United States: influence of hospital volume. Circ Arrhythm Electrophysiol 2015;8:42-8.

12. Healthcare Cost and Utilization Project. NIS related reports. Available online: https://www.hcup-us.ahrq.gov/ $\mathrm{db} /$ nation/nis/nisrelatedreports.jsp

13. Healthcare Cost and Utilization Project. HCUP methods series. 2002. Available online: https://www. hcup-us.ahrq.gov/reports/methods/1998Changesinthe NISRedesignFinal.pdf

Cite this article as: Solanki S, Haq KF, Chakinala RC, Khan Z, Aronow WS, Ali Khan M, Siddiqui MT, Haq KS, Frager S, Alimirah M, Nabors C, Samson DJ, Lebovics E, Wolf DC. Inpatient burden of esophageal varices in the United States: analysis of trends in demographics, cost of care, and outcomes. Ann Transl Med 2019;7(18):480. doi: 10.21037/atm.2019.08.34
14. Armitage P. Tests for Linear Trends in Proportions and Frequencies. Biometrics 1955;11:375-86.

15. Nahm FS. Nonparametric statistical tests for the continuous data: the basic concept and the practical use. Korean J Anesthesiol 2016;69:8-14.

16. Kim HJ, Fay MP, Feuer EJ, et al. Permutation tests for joinpoint regression with applications to cancer rates. Stat Med 2000;19:335-51.

17. Jamal MM, Samarasena JB, Hashemzadeh M, et al. Declining hospitalization rate of esophageal variceal bleeding in the United States. Clin Gastroenterol Hepatol 2008;6:689-95; quiz 605.

18. Hwang JH, Shergill AK, Acosta RD, et al. The role of endoscopy in the management of variceal hemorrhage. Gastrointest Endosc 2014;80:221-7.

19. Haq I, Tripathi D. Recent advances in the management of variceal bleeding. Gastroenterol Rep (Oxf) 2017;5:113-26.

20. D'Amico G, De Franchis R, Cooperative Study Group. Upper digestive bleeding in cirrhosis. Post-therapeutic outcome and prognostic indicators. Hepatology 2003;38:599-612.

21. El-Serag HB, Everhart JE. Improved survival after variceal hemorrhage over an 11-year period in the Department of Veterans Affairs. Am J Gastroenterol 2000;95:3566-73.

22. Pant C, Desai M, Deshpande A, et al. Esophageal variceal bleeding in hospitalized patients with cirrhosis. J Hosp Med 2015;10:453-6.

23. Thabut D, Hammer M, Cai Y, et al. Cost of treatment of oesophageal variceal bleeding in patients with cirrhosis in France: results of a French survey. Eur J Gastroenterol Hepatol 2007;19:679-86.

24. Volk ML, Tocco RS, Bazick J, et al. Hospital readmissions among patients with decompensated cirrhosis. Am J Gastroenterol 2012;107:247-52. 